\title{
SOME STRUCTURE THEORY FOR A CLASS OF TRIPLE SYSTEMS
}

\author{
BY
}

NORA C. HOPKINS

\begin{abstract}
This paper deals with a class of triple systems satisfying two generalized five linear identities and having nondegenerate bilinear forms with certain properties. If $(M,\{,\}$,$) is such a triple system with bilinear form \phi($,$) , it is shown that if$ $M$ is semisimple, then $M$ is the direct sum of simple ideals if $\phi$ is symmetric or symplectic or if $M$ is completely reducible as a module for its right multiplication algebra $\mathscr{L}$. It is also shown that if $M$ is a completely reducible $\mathscr{L}$-module, $M$ is the direct sum of a semisimple ideal and the center of $M$.

Such triple systems can be embedded into certain nonassociative algebras and the results on the triple systems are extended to these algebras.
\end{abstract}

1. Introduction. Suppose $(M,\{,\}$,$) is a finite dimensional triple system over a$ field $k$ of characteristic not two. Set $x R(y, z):=\{x y z\}$ and let $\mathscr{L}$ be the linear span of the $R(y, z)$ 's in End $M .(M,\{,\}$,$) is an inner derivation triple system if$

$$
\{\{x y z\} u v\}-\{\{x u v\} y z\}=\{x\{y u v\} z\}+\{x y\{z u v\}\}
$$

for all $x, y, z, u, v \in M$. (1.1) implies that $\mathscr{L}$ is a Lie subalgebra of $\operatorname{gl}(M)$ consisting of derivations of $(M,\{,\}$,$) , called inner derivations. More generally, (M,\{,\}$,$) is a$ twisted inner derivation triple system if there is a linear order one or two bijection $\tau$ on $L$ such that

$$
\{\{x y z\} u v\}-\{\{x u v\} y z\}=\left\{x\{y u v\}^{\prime} z\right\}+\{x y\{z u v\}\}
$$

and

$$
\left\{\{x y z\}^{\prime} u v\right\}^{\prime}-\left\{\{x u v\}^{\prime} y z\right\}^{\prime}=\left\{x\{y u v\}^{\prime} z\right\}^{\prime}+\{x y\{z u v\}\}^{\prime}
$$

for all $x, y, z, u, v \in M$, where $\{x y z\}^{\prime}:=x[R(y, z) \tau]$. (1.2) again implies that $\mathscr{L}$ is a Lie subalgebra of $\mathrm{gl}(M)$ and (1.3) implies that $\tau$ is a Lie algebra automorphism of $\mathscr{L}$. (1.2) and (1.3) reduce to (1.1) if $\tau=$ id.

Lie and anti-Lie triple systems are examples of inner derivation triple systems. Examples of twisted inner derivation triple systems (hereafter refered to as TIDTS) are Jordan triple systems and the ternary algebras considered in [1].

An ideal of a TIDTS $(M,\{,\}$,$) is a subspace N$ of $M$ such that $\{N M M\}+$ $\{M N M\}+\{M M N\}+\{N M M\}^{\prime}+\{M N M\}^{\prime}+\{M M N\}^{\prime} \subseteq N . N$ is abelian if $\{N N N\}+\{N N N\}^{\prime}=0 . N$ is central if $\{N M M\}+\{M N M\}+\{M M N\}=0$. If $N$ is central, $\{N M M\}^{\prime}+\{M N M\}^{\prime}+\{M M N\}^{\prime}=0$. The largest central ideal $Z(M)$

Received by the editors March 23, 1984.

1980 Mathematics Subject Classification. Primary 17A40, 17A60; Secondary 17B60, 17A70, 17 B70. 
of $(M,\{,\}$,$) is the center of (M,\{,\}) .,(M,\{,\}$,$) is simple if it has no ideals$ other than 0 and itself and $M$ is not abelian. A homomorphism from one TIDTS ( $M$, $\{,\}$,$) to another \left(M_{1},\{,,\}_{1}\right)$ is a linear mapping $\alpha: M \rightarrow M_{1}$ such that $\{x, y, z\} \alpha=\{x \boldsymbol{\alpha}, y \boldsymbol{\alpha}, z \boldsymbol{\alpha}\}_{1}$ and $\{x, y, z\}^{\prime} \boldsymbol{\alpha}=\{x \boldsymbol{\alpha}, y \alpha, z \boldsymbol{\alpha}\}_{1}^{\prime}$ for all $x, y, z \in M$. Clearly, ker $\alpha$ is an ideal of $M$.

Given a TIDTS $(M,\{,\}$,$) , a \mathbf{Z}_{2}$-graded algebra structure can be put on $\mathscr{S}(M):=M \oplus \mathscr{L}$ by setting

$$
\begin{gathered}
y z:=R(y, z) \\
x R(y, z):=-[R(y, z) \tau] x:=\{x y z\}
\end{gathered}
$$

and

$$
l_{1} l_{2}:=\left[l_{1}, l_{2}\right]
$$

for $x, y, z \in M, l_{1}, l_{2} \in \mathscr{L}$. Thus $\mathscr{S}(M)_{0}=\mathscr{L}$ and $\mathscr{S}(M)_{1}=M . \mathscr{S}(M)$ with this algebra structure is the standard embedding of $M$ and will be referred to simply as $\mathscr{S}$ when $M$ is understood. The algebra automorphism $\sigma$ of $\mathscr{S}$, defined by $(x+l) \sigma:=$ $-x+l$ for $x \in M, l \in \mathscr{L}$, is the standard involution of $\mathscr{S}$. Rewriting (1.2) and (1.3) in terms of the product on $\mathscr{S}$ gives

$$
(x(y z))(u v)-(x(u v))(y z)=x[y(z(u v))-((u v) y) z]
$$

$$
(u v)((y z) x)-(y z)((u v) x)=[((u v) y) z-y(z(u v))] x
$$

for $x, y, z, u, v \in \mathscr{S}_{1}$. It is easy to establish that $\mathscr{S}$ is a Lie algebra if and only if ( $M$, $\{,\}$,$) is a Lie triple system.$

Hereafter, $(M,\{,\}$,$) will denote a TIDTS over k$ with standard embedding $\mathscr{S}$.

If $N$ is an ideal of $(M,\{,\}$,$) , then the ideal of \mathscr{S}$ generated by $N$ is $\mathscr{I}(N):=N \oplus R(N, M)+R(M, N)$, where $R(N, M)+R(M, N)$ is the linear span of the $R(y, z)$ 's with $y \in N$ or $z \in N$, and $\mathscr{I}(N) \sigma \subseteq \mathscr{I}(N)$. Conversely, if $I$ is an ideal of $\mathscr{S}$ with $I \sigma \subseteq I$, then $I_{1}:=I \cap M$ is an ideal of $M$. The proof of the following proposition is the same as for Lie triple systems, see [4].

Proposition 1.9. (i) If $(M,\{,\}$,$) is simple, then \mathscr{S}$ is either simple or the direct sum of two simple ideals. In the latter case, $(M,\{,\}$,$) is a Lie triple system coming$ from a Lie algebra. Conversely, if $\mathscr{S}$ is simple, then $(M,\{,\}$,$) is simple.$

(ii) If $(M,\{,\}$,$) is the direct sum of simple ideals, then \mathscr{S}$ is the direct sum of simple ideals and conversely.

Proof. For the second statement of (i) note that if $I$ is a simple ideal of $\mathscr{S}$, then $I \cap I \sigma=0$ by the simplicity of $(M,\{,\}$,$) so \mathscr{S}=I \oplus I \sigma$ and $x \mapsto x-x \sigma$, $x \mapsto x+x \sigma$ are isomorphisms of $I$ to $M$ and $\mathscr{S}$ respectively.

If $N$ is an ideal of $(M,\{,\}$,$) , let N^{(0)}:=N$ and inductively define

$$
N^{(i)}:==\left\{N^{(i-1)} N^{(i-1)} N^{(i-1)}\right\}+\left\{N^{(i-1)} N^{(i-1)} N^{(i-1)}\right\}^{\prime}
$$

for $i \geqslant 1$. If $I$ is an ideal of $\mathscr{S}$, let $I^{(0)}:=I$ and

$$
I^{(i)}:=I^{(i-1)} I^{(i-1)}:=\left\{\sum_{i=1}^{n} a_{i} b_{i} \mid a_{i}, b_{i} \in I^{(i-1)}, n \in \mathbf{Z}^{+}\right\} \text {for } i \geqslant 1 .
$$


In general, for $i \geqslant 1 N^{(i)}$ is not an ideal of $(M,\{,\}$,$) and I^{(i)}$ is not an ideal of $\mathscr{S}$, but $N^{(i)}$ is a submodule of $M$ as an $\mathscr{L}$-module and if $I \sigma \subseteq I, I^{(i)}$ is a subalgebra of $\mathscr{S}$. If $N^{(i)}=0$ for some $i, N^{(i)}$ is solvable, and if $I^{(i)}=0$ for some $i$, then $I$ is solvable. Clearly, $N^{(i)} \subseteq \mathscr{I}(N)^{(i)}$ for every $i$ so if $I(N)$ is solvable, $N$ must be solvable. There is a unique maximal solvable ideal $\mathscr{R}(M)$ of $M$ and a unique maximal solvable ideal $\mathscr{R}(\mathscr{S})$ of $\mathscr{S}$, called the radical of $M$ and $\mathscr{S}$, respectively, see [5]. Clearly $\mathscr{R}(\mathscr{S}) \sigma \subseteq \mathscr{R}(\mathscr{S})$. If $\mathscr{R}(M)=0, M$ is semisimple and if $\mathscr{R}(\mathscr{S})=0, \mathscr{S}$ is semisimple. If $(M,\{,\}$,$) is simple, it is semisimple since M^{(1)}$ is an ideal of $(M,\{,\}$, and so $M^{(1)}=M$.

The proof of the following theorem is the same as for Lie triple systems [4].

THEOREM 1.10. (i) If $(M,\{,\}$,$) is semisimple, then \mathscr{S}$ is semisimple.

(ii) If $\mathscr{S}$ is semisimple, $Z(M)=0$.

In general a semisimple TIDTS is not the direct sum of simple ideals, see [3]. The purpose of the present paper is to show that this result is true for certain subclasses of TIDTS, namely twisted Lie module triple systems (see Definition 2.6) for which $\phi($,$) is symmetric or symplectic (Theorem 3.5) and also twisted Lie module triple$ systems which are completely reducible $\mathscr{L}$-modules (Theorem 4.3 ). For a completely reducible twisted Lie module triple system $(M,\{,\}$,$) it is also possible to show$ (Corollary 4.5) that $(M,\{,\}$,$) is reductive, i.e. the direct sum of a semisimple ideal$ and $Z(M)$. Then this gives the converse of Theorem 1.10(i) (Corollary 4.5). The problem of classifying completely reducible twisted Lie module triple systems is thus reduced to classifying the simple ones.

In $\$ 2$ twisted Lie module triple systems are defined and characterized and their standard embeddings are also characterized (Proposition 2.14). In \$3 some technical lemmas are proven as well as the desired result (Theorem 3.5) when $\phi($,$) is$ symmetric or symplectic. $\$ 4$ deals with completely reducible twisted Lie module triple systems.

I would like to thank John Faulkner for several helpful conversations and moral support.

2. Twisted Lie module triple systems. Suppose $\mathscr{L}$ is a finite dimensional Lie algebra over $k$ having a nondegenerate symmetric associative bilinear form (, ) and $\tau$ is an automorphism of $\mathscr{L}_{\text {such that }} \tau^{2}=$ id and

$$
\left(l \tau, l_{1} \tau\right)=\left(l, l_{1}\right)
$$

for all $l, l_{1} \in \mathscr{L}$. If $M$ is a faithful finite dimensional $\mathscr{L}$-module having a nondegenerate bilinear form $\phi($,$) such that$

$$
\phi(x l, y)+\phi(x, y(l \tau))=0
$$

for all $x, y \in M, l \in \mathscr{L}$, defining a bilinear mapping $R: M \times M \rightarrow \mathscr{L}$ by the condition

$$
(l, R(y, z))=\phi(z l, y)
$$

for every $l \in \mathscr{L}$ and trilinear mappings $\{,$,$\} and \{,,\}^{\prime}$ on $M$ by

$$
\{x y z\}:=x R(y, z)
$$


and

$$
\{x y z\}^{\prime}:=x[R(y, z) \tau]
$$

gives triple systems $(M,\{,\}$,$) and \left(M,\{,,\}^{\prime}\right)$.

Definition 2.6. If the triple system $(M,\{,\}$,$) can be constructed in the above$ fashion, it is a twisted Lie module triple system (TLMTS for short) and $\left(M,\{,\}^{\prime}\right)$ is dual to $(M,\{,\}$,$) . If \tau=\mathrm{id}, M$ is a Lie module triple system (LMTS for short).

If a new action of $\mathscr{L}$ on $M$ is defined by $x^{\prime} l:=x(l \tau)$, it is clear that $\left(M,\{,,\}^{\prime}\right)$ is also a TLMTS with dual $(M,\{,\}$,$) . TLMTS are a slight generalization of the triple$ systems introduced by Faulkner in [2] to study inner ideal geometries.

THEOREM 2.7. A triple system $(M,\{,\}$,$) is a TLMTS iff M$ has a trilinear product $\{,,\}^{\prime}$ and a nondegenerate bilinear form $\phi($,$) such that$

$$
\begin{gathered}
\{\{x y z\} u v\}-\{\{x u v\} y z\}=\left\{x\{y u v\}^{\prime} z\right\}+\{x y\{z u v\}\} \\
\phi(\{z u v\}, y)=-\phi\left(z,\{y u v\}^{\prime}\right) \\
\phi\left(\{z u v\}^{\prime}, y\right)=-\phi(z,\{y u v\}) \\
\phi(\{z u v\}, y)=\phi(\{v y z\}, u)
\end{gathered}
$$

and

$$
\phi\left(\{z u v\}^{\prime}, y\right)=\phi\left(\{v y z\}^{\prime}, u\right) .
$$

Proof. If $(M,\{,\}$,$) is a TLMTS with \{,,\}^{\prime}$ defined by (2.5), equations (2.7.2) and (2.7.3) are (2.2) with $l=R(u, v)$ and $l=[R(u, v) \tau]$ respectively, and (2.7.4) and (2.7.5) follow from the symmetry of (, ) since $(R(u, v) \tau, R(y, z))=$ $(R(y, z) \tau, R(u, v))$. For (2.7.1) note that in operator form it is

$$
[R(y, z), R(u, v)]=R\left(\{y u v\}^{\prime}, z\right)+R(y,\{z u v\}) .
$$

Now if $l, l_{1} \in \mathscr{L}$,

$$
\begin{aligned}
(l, R(y, z l)+R & (y(l \tau), z))=\phi\left(z l l_{1}, y\right)+\phi\left(z l_{1}, y(l \tau)\right) \\
& =\phi\left(z\left[l, l_{1}\right], y\right)=\left(\left[l, l_{1}\right], R(y, z)\right)=\left(l_{1},[R(y, z), l]\right)
\end{aligned}
$$

giving

$$
[R(y, z), l]=R(y(l \tau), z)+R(y, z l)
$$

which is $\left(2.7 .1^{\prime}\right)$ when $l=R(u, v)$.

Conversely, if $(M,\{,\}$,$) is a triple system having a triple product \{,,\}^{\prime}$ and a nondegenerate bilinear form $\phi($,$) statisfying (2.7.1)-(2.7.5), (2.7.1) implies the$ $R(y, z)$ 's span a subalgebra $\mathscr{L}$ of $\mathrm{gl}(M)$ with a bilinear form $($,$) defined by$ $(l, R(y, z)):=\phi(z l, y)$ for $l \in \mathscr{L}, y, z \in M$. (2.7.4) implies ( , ) is well defined and symmetric, and (, ) is nondegenerate since $\phi($,$) is nondegenerate and M$ is faithful. Defining $\tau: \mathscr{L} \rightarrow \mathscr{L}$ by

$$
(R(u, v) \tau, R(y, z)):=\phi\left(\{z u v\}^{\prime}, y\right)
$$

gives $(l \tau, R(y, z))=\phi(z(l \tau), y)$ for all $l \in L$, and (2.7.2) implies $\phi(z l, y)=$ $-\phi(z, y(l \tau))$ which is (2.2), while (2.7.3) implies $\phi(z(l \tau), y)=-\phi(z, y l)$. Hence $\phi\left(z\left(l \tau^{2}\right), y\right)=-\phi(z, y(l \tau))=\phi(z l, y)$ so $\tau^{2}=$ id and $\tau$ is bijective. By (2.7.5) 
$(R(u, v) \tau, R(y, z))=(R(y, z) \tau, R(u, v))$ giving $(2.1) .($,$) is associative by the$ argument for (2.7.6) in reverse using (2.7.1') and (2.7.2). For $l, l_{1} \in \mathscr{L}, y, z \in M$,

$$
\begin{aligned}
\left(\left[l, l_{1}\right] \tau, R(y, z)\right) & =\phi\left(z\left[l, l_{1}\right] \tau, y\right)=-\phi\left(z, y\left[l, l_{1}\right]\right) \\
& =-\phi\left(z, y l l_{1}\right)+\phi\left(z, y l_{1} l\right) \\
& =\phi\left(z\left[l \tau, l_{1} \tau\right], y\right)=\left(\left[l \tau, l_{1} \tau\right], R(y, z)\right)
\end{aligned}
$$

showing that $\tau$ is a Lie algebra automorphism, and $(M,\{\}$,$) is a TLMTS$ constructed using $\mathscr{L}, \tau,($, ), and $\phi($,$) .$

COROllary 2.8. A triple system $(M,\{,\}$,$) is a LMTS iff it has a nondegenerate$ bilinear form $\phi($, ) such that

$$
\begin{aligned}
\{\{x y z\} u v\}-\{\{x u v\} y z\} & =\{x\{y u v\} z\}+\{x y\{z u v\}\}, \\
\phi(\{z u v\}, y) & =-\phi(z,\{y u v\})
\end{aligned}
$$

and

$$
\phi(\{z u v\}, y)=\phi(\{v y z\}, u) .
$$

In operator form $(2.8 .1)$ is

$$
[R(y, z), R(u, v)]=R(\{y u v\}, z)+R(y,\{z u v\}) .
$$

COROllary 2.9. (i) If $(M,\{,\}$,$) is a TLMTS, it is a TIDTS.$

(ii) If $(M,\{,\}$,$) is a LMTS, it is an inner derivation triple system.$

EXAMPLE 2.10. For an example of a TIDTS which is not a TLMTS consider the Lie superalgebra $W(3)$ (defined in 3.1 .1 of [3]) which is the standard embedding of an anti-Lie triple system. In this case $\mathscr{L}$ has a Levi decomposition as $\mathscr{L}=\mathscr{L}^{\prime} \oplus$ $\operatorname{Rad} \mathscr{L}$, where $\mathscr{L}^{\prime}$ is a Lie algebra of type $A_{2}$ and $\operatorname{Rad} \mathscr{L}$ is four dimensional. By taking a Cartan subalgebra $H$ of $\mathscr{L}^{\prime}$ and computing the weights of $\mathscr{L}$ under the adjoint representation of $\mathscr{L}^{\prime}$, it is easy to check that there are weights $\mu$ whose negatives are not weights. But if $x_{\mu_{i}}$ is a weight vector of weight $\mu_{i}$ for $i=1,2$ and $h \in H$, then for any nondegenerate associative bilinear form ( , ) on $\mathscr{L}$

$$
\mu_{1}(h)\left(x_{\mu_{1}}, x_{\mu_{2}}\right)=\left(\left[x_{\mu_{1}}, h\right], x_{\mu_{2}}\right)=-\left(x_{\mu_{1}},\left[x_{\mu_{2}}, h\right]\right)=-\mu_{2}(h)\left(x_{\mu_{1}}, x_{\mu_{2}}\right),
$$

so $\left(x_{\mu_{1}}, x_{\mu_{2}}\right)=0$ unless $\mu_{1}=-\mu_{2}$. Thus $\mathscr{L}$ cannot have a nondegenerate associative bilinear form, and this anti-Lie triple system is not a TLMTS.

Corollary 2.11. Suppose $\mathscr{L}, \tau,(),$,$M , and \phi($,$) are used to construct the$ $\operatorname{TLMTS}(M,\{,\}$,$) . Then$

$$
\mathscr{L}=R(M, M)=\left\{\sum_{i=1}^{n} R\left(y_{i}, z_{i}\right) \mid y_{i}, z_{i} \in M, n \in \mathbf{Z}^{+}\right\} .
$$

Proof. By (2.7.6), $R(M, M)$ is an ideal of $\mathscr{L}$ and in the proof of Theorem 2.7 it is shown that $($,$) restricted to R(M, M)$ is nondegenerate. Hence if $R(M, M)^{\perp}:=\{l$ $\in \mathscr{L} \mid(l, R(y, z))=0$ for all $y, z \in M\}$, then $\mathscr{L}=R(M, M) \oplus R(M, M)^{\perp}$. But if $l \in R(M, M)^{\perp}$, then $0=(l, R(y, z))=\phi(z l, y)$ for all $y, z \in M$, so $l=0$ since $M$ is faithful and $\phi($,$) is nondegenerate.$ 
From now on $(M,\{,\}$,$) will be a TLMTS over k$ constructed using $\mathscr{L},(),, \tau$ and $\phi($,$) .$

The proof of the following lemma can be found in [2].

Lemma 2.12. Suppose $\varepsilon= \pm 1$. If $z \in M$ such that $\phi(z, y)=\varepsilon \phi(y, z)$ for all $y \in M$, then $R(y, z)=-\varepsilon R(z, y)$ for all $y \in M$. Hence if $(M,\{,\}$,$) is a LMTS,$ $\phi(y, z)=\phi(z, y)$ for all $y, z \in M$ implies $R(y, z)=-R(z, y)$ for all $y, z \in M$, and $\phi(y, z)=-\phi(z, y)$ for all $y, z \in M$ implies $R(y, z)=R(z, y)$ for all $y, z \in M$.

The converse of Lemma 2.12 holds if $M$ is the direct sum of irreducible $\mathscr{L}$ modules, none of which are trivial.

Turning now to the standard embedding $\mathscr{S}$ of $(M,\{,\}$,$) , a nondegenerate$ bilinear form $\beta($, ) can be defined on $\mathscr{S}$ by

$$
\beta\left(x+l, y+l_{1}\right):=\phi(x, y)+\left(l, l_{1}\right)
$$

for $x, y \in M, l, l_{1} \in \mathscr{L}$. Hence $\beta(a \sigma, b)=\beta(a, b \sigma)$ for all $a, b \in \mathscr{S}$, where $\sigma$ is the standard involution of $\mathscr{S}$.

PROPOSITION 2.14. A $\mathbf{Z}_{2}$-graded algebra $\mathscr{S}=\mathscr{S}_{1} \oplus \mathscr{S}_{0}$ having a bilinear form $\beta($, ) is the standard embedding of a TLMTS iff there is a linear mapping $\tau: \mathscr{S}_{0} \rightarrow \mathscr{S}_{0}$ such that

$$
\begin{gathered}
\mathscr{S}_{0}=\mathscr{S}_{1} \mathscr{S}_{1}, \\
a b=-(b \tau) a \text { for all } a \in \mathscr{S}_{1}, b \in \mathscr{S}_{0}, \\
(x(y z))(u v)-(x(u v))(y z)=x[y(z(u v))-((u v) y) z] \\
\left.\beta\right|_{\mathscr{S}_{1} \times \mathscr{S}_{1}} \text { is nondegenerate }
\end{gathered}
$$

and

$$
\beta(a b, c)=\beta(b, c a) \text { for all } a, b, c \in \mathscr{S} .
$$

Proof. If $\mathscr{S}$ is the standard embedding of the TLMTS $(M,\{,\}),,(2.14 .1)-(2.14 .4)$ are clear from the definition of $\mathscr{S}$ and $\beta($,$) . (2.14.5) is obtained by a case-by-case$ check using (2.2) and (2.3).

Conversely, suppose $\mathscr{S}=\mathscr{S}_{1} \oplus \mathscr{S}_{0}, \beta($, ), and $\tau$ satisfy (2.14.1)-(2.14.5). Defining triple products on $\mathscr{S}_{1}$ by $\{x y z\}:=x(y z)$ and $\{x y z\}^{\prime}=-(y z) x$ gives (2.7.1) by (2.14.3). $\beta($,$) is nondegenerate on \mathscr{S}_{1} \times \mathscr{S}_{1}$ by hypothesis and satisfies (2.7.2) since for $y, z, u, v \in \mathscr{S}_{1}$

$$
\begin{aligned}
\beta(\{z u v\}, y) & =\beta(z(u v), y)=-\beta([(u v) \tau] z, y) \\
& =-\beta(z, y[(u v) \tau]) \quad \text { by }(2.14 .5) \\
& =-\beta(z,-(u v) y)=-\beta\left(z,\{y u v\}^{\prime}\right) .
\end{aligned}
$$

(2.7.3) is similar and (2.7.4) holds since

$$
\begin{aligned}
\beta(\{z u v\}, y) & =\beta(z(u v), y)=\beta(u v, y z) \quad \text { by }(2.14 .4) \\
& =\beta(v,(y z) u)=-\beta(v, u[(y z) \tau]) \\
& =-\beta([(y z) \tau] v, u)=\beta(v(y z), y)=\beta(\{v y z\}, u) .
\end{aligned}
$$


A similar proof gives (2.7.5). Hence $\left(\mathscr{S}_{1},\{,\},\right)$ is a TLMTS by Theorem 2.7 and $\mathscr{S}_{0}=\left(\mathscr{S}_{1}, \mathscr{S}_{1}\right)$ by $(1.4)$ so $\mathscr{S}$ is the standard embedding of $\left(\mathscr{S}_{1},\{,\},\right)$.

COROLlaRY 2.15. A $\mathbf{Z}_{2}$-graded algebra $\mathscr{S}=\mathscr{S}_{1} \oplus \mathscr{S}_{0}$ having a bilinear form $\beta($, ) is the standard embedding of a LMTS iff it has the following properties:

$$
\begin{gathered}
\mathscr{S}_{0}=\mathscr{S}_{1} \mathscr{S}_{1}, \\
a b=-b a \text { for all } a \in \mathscr{S}, b \in \mathscr{S}_{0}, \\
(x(y z))(u v)-(x(u v))(y z)=x[(y(u v)) z+y(z(u v))] \\
\left.\beta\right|_{\mathscr{S}_{1} \times \mathscr{S}_{1}} \text { is nondegenerate }
\end{gathered}
$$

and

$$
\beta(a b, c)=\beta(b, c a) \text { for all } a, b, c \in \mathscr{S}_{1} .
$$

3. Ideals and structure theory of TLMTS. We turn now to the ideals of a TLMTS $(M,\{,\}$,$) and its standard embedding \mathscr{S}$. Define Ann $\mathscr{L}$ to be Ann $\mathscr{L}=\{x \in$ $M \mid x l=0$ for all $l \in \mathscr{L}\}$.

LeMma 3.1. $Z(M)=$ Ann $\mathscr{L}$.

Proof. By Corollary $2.11 Z(M) \subseteq$ Ann $\mathscr{L}$. Now suppose $x \in$ Ann $\mathscr{L}, y \in M$, and $l \in \mathscr{L}$. Then by $(2.3),(l, R(y, x))=\phi(x l, y)=0$ so $R(y, x)=0$ and similarly $R(x, y)=0$. Hence $x \in Z(M)$.

Lemma 3.2. Suppose $M=N \oplus M_{1}$ as an $\mathscr{L}$-module with $N$ an ideal of $M$. Then

(i) $\left\{M_{1} N M\right\}+\left\{M_{1} M N\right\}+\left\{M_{1} N M\right\}^{\prime}+\left\{M_{1} M N\right\}^{\prime}=0$,

(ii) $R(N, M)+R(M, N)=R(N, N)$ and $R\left(N, M_{1}\right)=R\left(M_{1}, N\right)=0$,

(iii) $\mathscr{L}=R(N, N)+R\left(M_{1}, M_{1}\right)$, and

(iv) $\{M N M\}+\{M M N\}+\{M N M\}^{\prime}+\{M M N\}^{\prime} \subseteq N^{(1)}$.

Proof. (i) $\left\{M_{1} N M\right\}+\left\{M_{1} M N\right\}+\left\{M_{1} N M\right\}^{\prime}+\left\{M_{1} M N\right\}^{\prime} \subseteq M_{1} \cap N=0$.

(ii) $\mathscr{L}=R(N, M)+R(M, N)+R\left(M_{1}, M_{1}\right)=R(N, N)+R\left(N, M_{1}\right)+$ $R\left(M_{1}, N\right)+R\left(M_{1}, M_{1}\right)$. By (i)

$$
\left(R(N, M)+R(M, N), R\left(N, M_{1}\right)\right)=\phi\left(\left\{M_{1} N M\right\}+\left\{M_{1} M N\right\}, N\right)=0
$$

and

$$
\left(R\left(M_{1}, M_{1}\right), R\left(N, M_{1}\right)\right)=\left(R\left(N, M_{1}\right), R\left(M_{1}, M_{1}\right)\right)=\phi\left(\left\{M_{1} N M_{1}\right\}, M_{1}\right)=0
$$

so $R\left(N, M_{1}\right)=0$ by the nondegeneracy of (, ). Also by (i)

$$
\begin{aligned}
\left(R(N, M)+R(M, N), R\left(M_{1}, N\right)\right) & =\phi\left(\{N N M\}+\{N M N\}, M_{1}\right) \\
& =\phi\left(N,\left\{M_{1} N M\right\}^{\prime}+\left\{M_{1} M N\right\}^{\prime}\right)=0
\end{aligned}
$$

and $\left(R\left(M_{1}, M_{1}\right), R\left(M_{1}, N\right)\right)=\left(R\left(M_{1}, N\right), R\left(M_{1}, M_{1}\right)\right)=\phi\left(\left\{M_{1} M_{1} N\right\}, M_{1}\right)=0$ so $R\left(M_{1}, N\right)=0$.

(iii) follows from (ii) and (iv) from (i) and (ii). 
Lemma 3.3. Suppose $N$ is an ideal of $(M,\{,\}$,$) such that \phi($,$) restricted to N \times N$ is nondegenerate. Then $(N,\{,\}$,$) is a TLMTS and \mathscr{L}=R(N, N) \oplus$ Ann $N$, where Ann $N=\{l \in \mathscr{L} \mid x l=0$ for all $x \in N\}$.

Proof. The first statement follows from Theorem 2.7 and the second has the same proof as Corollary 2.11.

LEMMA 3.4. (i) If $I$ is an ideal of $\mathscr{S}$ such that $\beta(a, b)=0$ for all $a, b \in I, I^{(1)}=0$ so I is solvable.

(ii) If $N$ is an ideal of $(M,\{,\}$,$) such that \phi(x, y)=0$ for all $x, y \in N$, $\{N N M\}+\{N M N\}+\{N N M\}^{\prime}+\{N M N\}^{\prime}=0$ so $N$ is abelian and hence solvable. Also $R(N, M)+R(M, N)$ is an abelian ideal of $\mathscr{L}$.

Proof. (i) Suppose $a, b \in I$ and $c \in \mathscr{S}$. Then $\beta(a b, c)=\beta(b, c a)=0$ by (2.14.5) since $c a \in I$. Hence $a b=0$ by the nondegeneracy of $\beta($, ).

(ii) follows from (i) since if $\phi(x, y)=0$ for all $x, y \in N, \beta(a, b)=0$ for all $a, b \in \mathscr{I}(N)$.

THEOREM 3.5. Suppose $\phi($, ) is symmetric or symplectic.

(i) If $\mathscr{S}$ is semisimple, then $\mathscr{S}$ is the direct sum of simple ideals.

(ii) If $(M,\{,\}$,$) is semisimple, then (M,\{,\}$,$) is the direct sum of simple ideals$ which are themselves TLMTS.

Proof. (i) This is the usual Dieudonné argument: Let $I$ be an ideal of $\mathscr{S}$ minimal with respect to the property $I \sigma=I$. Then $I$ is either a minimal ideal of $\mathscr{S}$ or the direct sum of two minimal ideals. Now $I^{\perp}:=\{a \in \mathscr{S} \mid \beta(a, b)=0$ for all $b \in I\}$ is an ideal of $\mathscr{S}$ and $I^{\perp} \sigma=I^{\perp}$. Hence either $I \cap I^{\perp}=0$ or $I \cap I^{\perp}=I$. If $I \cap I^{\perp}=I$, $I$ is solvable by Lemma 3.4(i), contradicting the semisimplicity of $\mathscr{S}$. Thus $I \cap I^{\perp}=0$, $\mathscr{S}=I \oplus I^{\perp}$, and $\beta($,$) restricted to I \times I$ and $I^{\perp} \times I^{\perp}$ is nondegenerate. Hence $I$ and $I^{\perp}$ are the standard embeddings of the TLMTS $I \cap M$ and $I^{\perp} \cap M$, respectively, by Proposition 2.14 and the fact that $I I^{\perp}+I^{\perp} I \subseteq I \cap I^{\perp}=0$. And since any ideal of $I$ is an ideal of $\mathscr{S}, I$ is simple or the direct sum of simple ideals. Induction on $\operatorname{dim} \mathscr{S}$ finishes the proof.

(ii) This follows from (i), Proposition 1.9(ii) and Lemma 3.3.

4. Completely reducible TLMTS. In order to derive further results about a TLMTS $(M,\{,\}$,$) over k$, it is necessary to assume that $M$ is completely reducible as a module for $\mathscr{L}$. Conditions on $(M,\{,\}$,$) that imply M$ completely reducible have not yet been established. Recall that $\mathscr{R}=\mathscr{R}(M)$ is the solvable radical of $(M$, $\{,\}$,$) .$

Lemma 4.1. Suppose $M=M_{1} \oplus \mathscr{R}$ as an L-module. Then

(i) $\left\{M_{1} M_{1} M_{1}\right\}=M_{1}=\left\{M_{1} M_{1} M_{1}\right\}^{\prime}$,

(ii) $\phi\left(\mathscr{R}, M_{1}\right)=0=\phi\left(M_{1}, \mathscr{R}\right)$ and $\left.\phi\right|_{\mathscr{R} \times \mathscr{R}}$ and $\left.\phi\right|_{M_{1} \times M_{1}}$ are nondegenerate,

(iii) $\left\{\mathscr{R} M_{1} M_{1}\right\}=0=\left\{\mathscr{R} M_{1} M_{1}\right\}^{\prime}$, and

(iv) $M_{1}$ is a semisimple ideal of $M$ which is a TLMTS.

Proof. By Lemma 5.2, $\left\{M_{1} M M\right\}=\left\{M_{1} M_{1} M_{1}\right\}$. Then decomposing $M_{1}$ as an $\mathscr{L}$-module as $M_{1}=\left\{M_{1} M_{1} M_{1}\right\} \oplus M_{2}$ gives $\left\{M_{2} M M\right\} \subseteq M_{2} \cap\left\{M_{1} M_{1} M_{1}\right\}=0$ so 
$M_{2} \subseteq Z(M)$ by Lemma 3.1. But $Z(M) \subseteq \mathscr{R}$ so $M_{2} \subseteq M_{1} \cap \mathscr{R}=0$ and $M_{1}=$ $\left\{M_{1} M_{1} M_{1}\right\}$. The proof that $M_{1}=\left\{M_{1} M_{1} M_{1}\right\}^{\prime}$ is similar.

(ii) $\phi\left(M_{1}, \mathscr{R}\right)=\phi\left(\left\{M_{1} M_{1} M_{1}\right\}, \mathscr{R}\right)=\left(R\left(M_{1}, M_{1}\right), R\left(\mathscr{R}, M_{1}\right)\right)=0$ by Lemma 3.2(ii) and

$$
\begin{aligned}
\phi\left(\mathscr{R}, M_{1}\right) & =\phi\left(\mathscr{R},\left\{M_{1} M_{1} M_{1}\right\}\right)=\phi\left(\left\{\mathscr{R} M_{1} M_{1}\right\}^{\prime}, M_{1}\right) \\
& =\left(R\left(M_{1}, M_{1}\right) \tau, R\left(M_{1} \mathscr{R}\right)\right)=0,
\end{aligned}
$$

again by Lemma 3.2(ii). The nondegeneracy of $\phi($, ) then implies the nondegeneracy of $\left.\phi\right|_{\mathscr{R} \times \mathscr{R}}$ and $\left.\phi\right|_{M_{1} \times M_{1}}$.

(iii) By Lemma 3.2(i) and (2.7.4) $0=\phi\left(\left\{M_{1} \mathscr{R} \mathscr{R}\right\}, M_{1}\right)=\phi\left(\left\{\mathscr{R} M_{1} M_{1}\right\}, \mathscr{R}\right)$ so $\left\{\mathscr{R} M_{1} M_{1}\right\}=0$ by the nondegeneracy of $\left.\phi\right|_{\mathscr{R} \times \mathscr{R}}$. That $\left\{\mathscr{R} M_{1} M_{1}\right\}^{\prime}=0$ follows from Lemma 3.2(i) and (2.7.5).

(iv) $M_{1}$ is an ideal by (iii) and Lemma 3.2. Since any ideal of $M_{1}$ is an ideal of $(M,\{,\}$,$) , the solvable radical of M_{1}$ is contained in $\mathscr{R}$ and so must be 0 . Hence $M_{1}$ is semisimple. It is a TLMTS by (ii) and Lemma 3.3.

Lemma 4.2. Suppose $N$ is an ideal of $(M,\{,\}$,$) . Then N^{(k)}$ is an ideal for every $k$.

Proof. $N^{(1)}$ is a submodule by (1.2) and (1.3) and hence is an ideal by Lemma 3.2(iv). That $N^{(k)}$ is an ideal for every $k$ now follows by induction on $k$.

TheOREM 4.3. Suppose $(M,\{,\}$,$) is semisimple. Then M$ is the direct sum of simple ideals which are TLMTS.

Proof. Suppose $N$ is a minimal ideal of $M$. Then $M=N \oplus M_{1}$ as an $\mathscr{L}$-module with $\mathscr{L}=R(N, N) \oplus R\left(M_{1}, M_{1}\right)$ by Lemma 3.2. $N^{(1)}=N$ since otherwise $N^{(1)}=0$ by Lemma 4.2 and the minimality of $N$, contradicting the semisimplicity of $(M$, $\{$, , $\})$. Hence $\phi\left(N, M_{1}\right)=\phi\left(\{N N N\}, M_{1}\right)=\phi\left(N,\left\{M_{1} N N\right\}^{\prime}\right)=0$ by Lemma 3.2(i) and similarly $\phi\left(M_{1}, N\right)=0$. Thus $\left.\phi\right|_{N \times N}$ and $\left.\phi\right|_{M_{1} \times M_{1}}$ are nondegenerate and $(N,\{,\}$,$) is a TLMTS by Lemma 3.3. Also, since$

$$
\left(R(N, N), R\left(M_{1}, M_{1}\right)\right)=\phi\left(\left\{M_{1} N N\right\}, M_{1}\right)=0
$$

by Lemma 3.2(i), ( , ) $\left.\right|_{R(N, N) \times R(N, N)}$ and ( , ) $\left.\right|_{R\left(M_{1}, M_{1}\right) \times R\left(M_{1}, M_{1}\right)}$ are nondegenerate. $\phi\left(\left\{N M_{1} M_{1}\right\}, N\right)=\left(R\left(M_{1}, M_{1}\right), R(N, N)\right)=0$ so $M_{1}$ is an ideal of $M$ by Lemma 3.2(i) and is a TLMTS by Lemma 3.3. Since any ideal of $\left(M_{1},\{,\},\right)$ is an ideal of $M, M_{1}$ is semisimple and induction on $\operatorname{dim} M$ gives $M_{1}$ as a direct sum of simple ideals which are TLMTS. $N$ is simple since it is minimal and any ideal of $N$ is an ideal of $(M,\{,\}$,$) .$

THEOREM 4.4. If $(M,\{,\}$,$) is solvable, then it is abelian.$

Proof. There is a $k$ such that $M^{(k)} \neq 0$ and $M^{(k+1)}=0$. The result is true if $k=0$, so suppose by way of contradiction that $k>0$. Then $M^{(k)}$ is an abelian ideal of $M$ by Lemma 4.2 and decomposing $M$ as an $\mathscr{L}$-module as $M=N \oplus M^{(k)}$ gives $\mathscr{L}$ $=R(N, N)+R\left(M^{(k)}, M^{(k)}\right)$ and $\left\{N M^{(k)} M^{(k)}\right\}=0$ by Lemma 3.2. Hence $R\left(M^{(k)}, M^{(k)}\right)=R\left(M, M^{(k)}\right)+R\left(M^{(k)}, M\right)=0$ and $\mathscr{L}=R(N, N)$. Also $\phi\left(\left\{M^{(k)} N N\right\}, M\right)=\left(R(N, N), R\left(M, M^{(k)}\right)\right)=0$ so $\left\{M^{(k)} N N\right\}=0$ and $N$ is an 
ideal of $(M,\{,\}$,$) . Thus M^{(1)}=N^{(1)}+M^{(k)(1)}=N^{(1)}+M^{(k+1)}=N^{(1)}$ and so $M^{(i)}=N^{(i)}$ for $i \geqslant 1$. But then $M^{(k)} \subseteq N^{(k)} \subseteq M^{(k)} \cap N=0$ contradicting the choice of $k$ and giving the desired result.

COROLlaRy 4.5. (i) $(M,\{,\}$,$) is reductive.$

(ii) $(M,\{,\}$,$) is semisimple if and only if \mathscr{S}$ is semisimple. If $(M,\{,\}$,$) is$ semisimple, both $(M,\{,\}$,$) and \mathscr{S}$ are the direct sum of simple ideals.

Proof. (i) This follows from Lemma 4.1 and Theorem 4.4.

(ii) The first statement follows from (i) and Theorem 1.10. The second statement follows from Theorem 4.3 and Proposition 1.9(ii).

By Corollary 4.5 the problem of classifying completely reducible TLMTS is now reduced to classifying simple ones.

\section{REFERENCES}

1. J. R. Faulkner, A construction of Lie algebras from a class of ternary algebras, Trans. Amer. Math. Soc. 155 (1971), 397-408.

2. On the geometry of inner ideals, J. Algebra 26 (1973), 1-9.

3. V. G. Kac, Lie superalgebras, Adv. in Math. 26 (1977), 8-96.

4. W. G. Lister, A structure theory of Lie triple systems, Trans. Amer. Math. Soc. 72 (1952), 217-242.

5. K. Meyberg, Lectures on algebras and triple systems, Lecture Notes, Univ. of Virginia, Charlottesville, Va., 1972.

Department of Mathematics, Iowa State University, Ames, Iowa 50011

Current address: Department of Mathematics, The Ohio State University, Columbus, Ohio 43210-1174 